\title{
In-situ characterization of O-terminated Cu tips for high-resolution atomic force microscopy
}

Cite as: Appl. Phys. Lett. 114, 143103 (2019); https://doi.org/10.1063/1.5085747

Submitted: 14 December 2018 . Accepted: 25 March 2019. Published Online: 10 April 2019

Alexander Liebig (D), and Franz J. Giessibl (D)

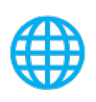

View Online

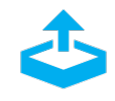

Export Citation

\section{ARTICLES YOU MAY BE INTERESTED IN}

The qPlus sensor, a powerful core for the atomic force microscope

Review of Scientific Instruments 90, 011101 (2019); https://doi.org/10.1063/1.5052264

Evaluating the potential energy landscape over single molecules at room temperature with lateral force microscopy

Applied Physics Letters 112, 181601 (2018); https://doi.org/10.1063/1.5026671

Switching dynamics of ferroelectric $\mathrm{HfO}_{2}-\mathrm{ZrO}_{2}$ with various $\mathrm{ZrO}_{2}$ contents

Applied Physics Letters 114, 142902 (2019); https://doi.org/10.1063/1.5093793

\section{Applied Physics Reviews} Now accepting original research 


\title{
In-situ characterization of O-terminated Cu tips for high-resolution atomic force microscopy
}

\author{
Cite as: Appl. Phys. Lett. 114, 143103 (2019); doi: 10.1063/1.5085747 \\ Submitted: 14 December 2018 - Accepted: 25 March 2019 . \\ Published Online: 10 April 2019
}

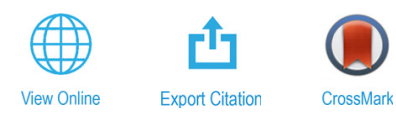

\author{
Alexander Liebig $^{a)}$ (D) and Franz J. Giessibl ${ }^{\mathrm{b})}$ (D) \\ AFFILIATIONS \\ Institute of Experimental and Applied Physics, University of Regensburg, D-93040 Regensburg, Germany \\ a)alexander.liebig@ur.de \\ b) franz.giessibl@ur.de
}

\begin{abstract}
Functionalizing a metal tip with a single CO molecule (CO tip) leads to an unprecedented spatial resolution of small organic molecules by frequency-modulation atomic force microscopy (FM-AFM) at low temperatures. O-terminated Cu tips (CuOx tips) show comparable imaging capabilities as $\mathrm{CO}$ tips but exhibit a much stiffer apex. So far, to verify tip functionalization with oxygen (i.e., CuOx tips), scanning tunneling microscopy and AFM images, together with force spectroscopy curves of copper oxide domains, have been compared with calculated data for different tip models. Here, we apply the carbon-monoxide front atom identification (COFI) method and additional force spectroscopy to characterize $\mathrm{CuOx}$ tips in-situ on a $\mathrm{Cu}(110)$ surface. In $\mathrm{COFI}$, a single $\mathrm{CO}$ molecule adsorbed on a $\mathrm{Cu}$ surface is imaged to atomically resolve the tip apex. Based on our findings, we suggest accompanying tip fingerprinting with COFI and force spectroscopy to identify the atomic and chemical compositions of the apex of CuOx tips for high-resolution AFM experiments.
\end{abstract}

Published under license by AIP Publishing. https://doi.org/10.1063/1.5085747

The spatial contrast in scanning tunneling microscopy (STM) and atomic force microscopy $(\mathrm{AFM})^{2}$ depends crucially on the exact geometrical structure and chemical species of the tip apex. ${ }^{3}$ The spatial resolution can be increased by controlled modification of the tip apex. In STM, it was found that by trapping a single hydrogen molecule in the tip-sample junction, the internal structure of a molecule adsorbed on a surface can be resolved. ${ }^{4}$ Using vertical manipulation, ${ }^{5}$ individual atoms and molecules can be picked up with an STM tip to functionalize the tip apex. ${ }^{6,7}$ CO-terminated metal tips (CO tips), which can be prepared by picking up a single carbon monoxide molecule from a $\mathrm{Cu}$ surface, ${ }^{6}$ enable submolecular resolution imaging of organic molecules and bond-order discrimination by AFM. ${ }^{8,9}$ Terminating the tip apex with noble gas atoms achieves a similar spatial resolution. ${ }^{10}$ Although functionalizing the tip with a $\mathrm{CO}$ molecule yields an increase in the spatial resolution, interpretation of atomic-scale contrast with these tips is highly nontrivial: When the tip interacts with the sample, lateral forces cause lateral deflection of the $\mathrm{CO}$ molecule that can create artifacts in the images and hence inhibit a direct interpretation of data. ${ }^{9,11-14}$ Recently, Mönig and co-workers proposed using oxygenterminated $\mathrm{Cu}$ tips (CuOx tips) that show a comparable spatial resolution and much higher lateral stiffness of the tip apex. ${ }^{15,16}$ With such $\mathrm{CuOx}$ tips, it is for example possible to quantitatively measure intramolecular bond lengths, ${ }^{16}$ which appear elongated when using $\mathrm{CO}$ tips, ${ }^{9}$ and to scan with video rate imaging velocities theoretically.
While the functionalization of a metal tip with a $\mathrm{CO}$ molecule is a well-controlled process, ${ }^{6} \mathrm{CuOx}$ tips are prepared by repeated collisions of the tip with an oxidized $\mathrm{Cu}$ surface. ${ }^{15}$ This is in general a random process that involves many atoms of both the tip and the surface, which means that the exact atomic structure and the chemical species of the atoms composing the tip apex after the collision are not known. Knowledge of the chemical and structural identities of the tip is of crucial importance, especially when experiments are accompanied by theoretical calculations involving the tip structure and its chemical composition, and hence, these properties should be well-characterized in the experiment. So far, the verification of tip functionalization with oxygen relies on comparing experimental results with calculations: The experimental STM and AFM images of copper oxide domains are compared with density functional theory (DFT) and nonequilibrium Greens function (NEGF) calculations to determine the chemical identity of the tip apex. ${ }^{18-21}$ A description of the atomic tip apex structure requires a large tip model database obtained after extensive and timeconsuming simulations, and precise determination of the structural apex composition is not always possible. ${ }^{2}$

Conversely, a carbon-monoxide molecule adsorbed on a $\mathrm{Cu}(111)$ surface can be used to image the tip apex with atomic resolution, a technique known as the carbon-monoxide front atom identification (COFI) method. ${ }^{22-24}$ In COFI, the tip is scanned at a constant height above the $\mathrm{CO}$ molecule which acts as a probe, and the resulting images 
reveal the atomic configuration of the tip apex [compare Fig. 1(a)]. Additionally, the chemical species of atoms can be distinguished by force spectroscopy. Sugimoto and co-workers ${ }^{25}$ showed that the chemical species of different surface atoms can be determined by analyzing the minimum of the short-range interaction between the tip and the individual surface atoms. By knowing the chemical species on the surface, this concept can be transferred to determine the chemical species of the tip apex atom: Hofmann and co-workers ${ }^{26}$ distinguished between the chemical species of the frontmost atom of different single-atom metal tips using force spectroscopy above the same chemical species on the surface, i.e., a single CO molecule adsorbed on $\mathrm{Cu}(111)$.

In this Letter, we present experimental characterization of $\mathrm{CuOx}$ tips by a combination of COFI and force spectroscopy on a $\mathrm{Cu}(110)$ surface. In this way, both the structural and the chemical compositions of the tip apices can be determined in-situ.

The experiments were carried out on a commercial lowtemperature scanning tunneling/atomic force microscope operating at a temperature of $4.4 \mathrm{~K}$ (LT STM/AFM, Scienta Omicron GmbH, Taunusstein). We used a qPlus sensor ${ }^{27}$ equipped with an electrochemically etched tungsten tip, with a resonance frequency of $f_{0}=$ $46597 \mathrm{~Hz}$, a stiffness of $k=1800 \mathrm{Nm}^{-1}$, and a quality factor of $Q=482321$. The sensor was operated at a constant amplitude of $A=50 \mathrm{pm}$ in the frequency-modulation mode (FM-AFM). ${ }^{28}$ Except when explicitly mentioned, all data presented here are raw data.

The $\mathrm{CuOx}$ tips were characterized and prepared following the procedure described by Mönig and co-workers. ${ }^{15}$ Upon low oxygen coverages, $\mathrm{Cu}(110)$ undergoes a $(2 \times 1)$-O added-row $(\mathrm{AR})$ reconstruction [shown in Figs. 1(b) and 1(c)], forming striped copper oxide domains along the [001] direction. ${ }^{15,29-32}$ To functionalize the tip apex with oxygen, the tip is repeatedly dipped into the oxide domains until a notable enhancement in corrugation within the oxide is observed: Fig. 1(c) shows an STM image recorded in the constant-current mode with a bare metal tip, resolving the copper oxide domains as darker regions than the clean $\mathrm{Cu}$ surface. For comparison, in Fig. 2(d), an STM image of the AR reconstruction recorded with a $\mathrm{CuOx}$ tip is shown: Here, the copper oxide domain shows a larger corrugation (see line profiles in Fig. S1 in the supplementary material), which indicates a change in the chemical species of the tip terminating atom. ${ }^{21}$ In order to characterize the tips using the COFI method, we additionally dosed approximately $0.005 \mathrm{ML}$ of carbon-monoxide on the surface [see Fig. 1(c)]. It should be noted that the COFI method exploits the vertical on-top adsorption configuration of individual $\mathrm{CO}$ molecules on $\mathrm{Cu}(111)$ with the $\mathrm{O}$ atom pointing away from the surface. Here, we use $\mathrm{COFI}$ on a $\mathrm{Cu}(110)$ surface, where $\mathrm{CO}$ also adsorbs in this vertical on-top configuration as determined by photoelectron diffraction. ${ }^{33}$

The interaction of a $\mathrm{CuOx}$ tip with a $\mathrm{CO}$ molecule adsorbed on $\mathrm{Cu}(110)$ as a function of tip-sample distance is comparable to the findings of Sun et al. for a CO tip on $\mathrm{CO} / \mathrm{Cu}(111) .{ }^{11}$ Figure $2(\mathrm{~b})$ shows frequency shift vs distance $\Delta f(z)$ spectra recorded with a CuOx tip above the $\mathrm{CO}$ molecule and above the bare $\mathrm{Cu}(110)$ surface (red and black curves, respectively). The zero point of the experimental $z$ axis defines the closest tip-sample approach in the measurements with this tip. Approaching the surface from $z=1 \mathrm{~nm}$, the $\Delta f$ values are initially more negative above the $\mathrm{CO}$ molecule as compared to the bare $\mathrm{Cu}$ surface, which we attribute to van der Waals attraction between the tip and the $\mathrm{CO}$ molecule. Upon further approach, the spectrum above the $\mathrm{CO}$ reaches a minimum and becomes less negative, which can be assigned to stronger contribution of short-range Pauli repulsion between the tip and the $\mathrm{CO}$ molecule. At this point of maximum contrast $z_{\mathrm{COFI}}$, we record a constant-height AFM image [a COFI image, Fig. 2(a)] of the CuOx tip, resolving a single bright, circularly symmetric feature with a dark ring around it. We show COFI images of our $\mathrm{CuOx}$ tip as a function of tip-sample distance in the supplementary material to further illustrate this contrast evolution (see Fig. S2).

The short-range component of the interaction between the tip and the $\mathrm{CO}$ molecule can be obtained by performing the "on-off" subtraction method. ${ }^{11,26,36}$ We subtracted the $\Delta f(z)$ spectrum recorded above the bare $\mathrm{Cu}(110)$ surface from the spectrum recorded above the $\mathrm{CO}$ molecule and calculated the short-range force $F_{\mathrm{SR}}(z)$ via the Sader-Jarvis force deconvolution method [compare Fig. 2(c)]. ${ }^{34}$ The force deconvolution has been explicitly checked for well-posed behavior. ${ }^{37} \mathrm{We}$ obtain a short-range force minimum of $-15 \mathrm{pN}$ for the $\mathrm{CuOx}$ tip. In the supplementary material, we present $F_{\mathrm{SR}}(z)$ spectra for twelve different $\mathrm{CuOx}$ tips, which all exhibit short-range force minima between $-15 \mathrm{pN}$ and $-30 \mathrm{pN}$ (see Fig. S3). For comparison, we furthermore show a COFI image and $F_{\mathrm{SR}}(z)$ spectra of a COterminated tip recorded on $\mathrm{CO} / \mathrm{Cu}(110)$ in the supplementary material (see Fig. S4), also exhibiting a short-range force minimum of -15 $\mathrm{pN}$. The values we obtained for both the $\mathrm{CuOx}$ and $\mathrm{CO}$ tips are close to the value obtained with a $\mathrm{CO}$ tip on $\mathrm{Cu}(111)^{11}$ and show that oxidizing the tip apex effectively reduces the chemical reactivity: ${ }^{15}$ short- (a)

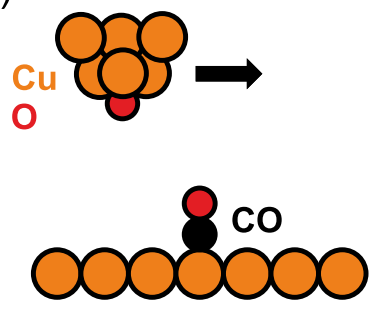

(b)

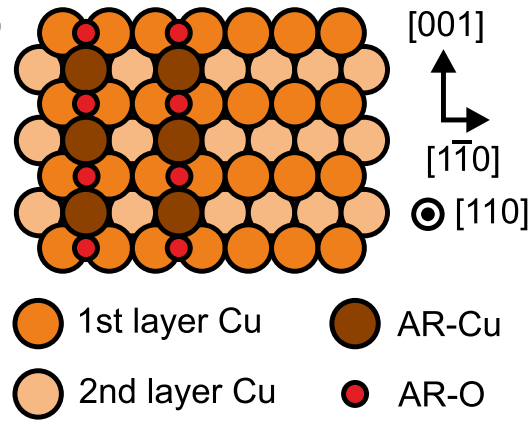

(c)

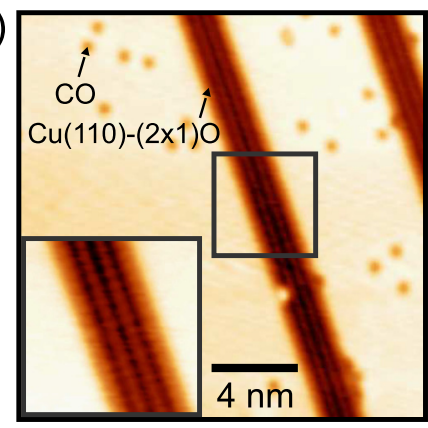

$68 \mathrm{pm}$

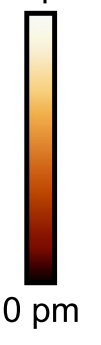

FIG. 1. (a) Schematic of the COFI method: An AFM tip, in this case, a CuOx tip, is scanned at a relatively close distance at a constant height above a CO molecule adsorbed on the $\mathrm{Cu}(110)$ surface. (b) Top view of the $\mathrm{Cu}(110)-(2 \times 1) \mathrm{O}$ added-row (AR) reconstruction. ${ }^{15}$ (c) STM overview image of the $\mathrm{Cu}(110)$ surface recorded with a metal tip. Dark, striped regions represent the copper oxide domains on $\mathrm{Cu}(110)$. Single, dark points are individual $\mathrm{CO}$ molecules adsorbed on the bare $\mathrm{Cu}(110)$ surface. Imaging parameters: sample bias $V_{b}=-100 \mathrm{mV}$ tunneling current setpoint $\left\langle\mathrm{I}_{\mathrm{t}}\right\rangle=-100 \mathrm{pA}$. Inset: Zoom-in of the area marked by the square. 
range force minima of metal tips on $\mathrm{CO} / \mathrm{Cu}(111)$ are typically lower than $-130 \mathrm{pN}^{2}$

We then imaged the $\mathrm{Cu}(110)-(2 \times 1) \mathrm{O}$ reconstruction with the $\mathrm{CuOx}$ tip, as has been done in previous studies in which STM and AFM images alongside DFT-based calculations were used to determine the tip apex. ${ }^{15,16}$ In Fig. 2(d), an STM topography image of the copper oxide domain is shown, where the maxima within the oxide coincide with AR positions in good agreement with Ref. 16. (Interestingly, the maxima were found between the added rows in Ref. 15, possibly due to the different STM imaging heights, ${ }^{21}$ as discussed in section S1 in the supplementary material.) (a)
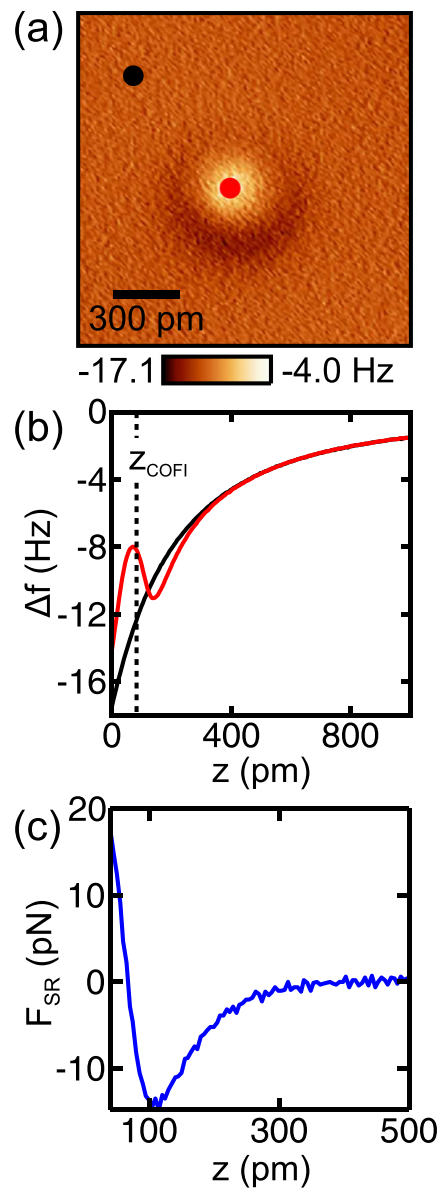

(d)

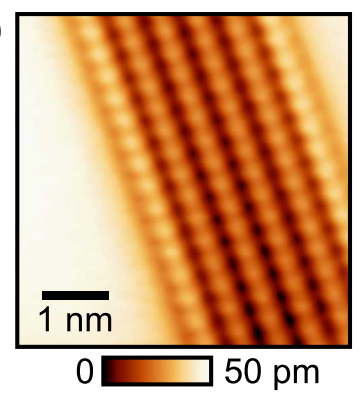

(e)

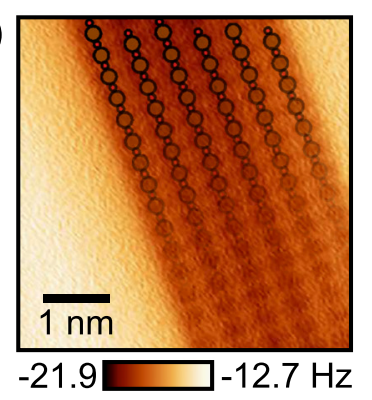

(f)

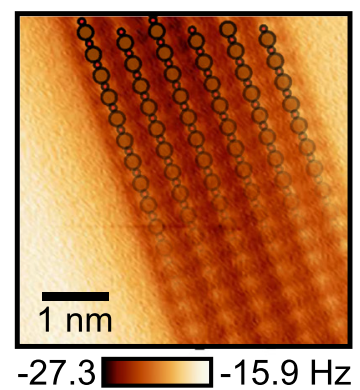

FIG. 2. (a) COFI image of a CuOx tip. (b) $\Delta f(z)$ spectra recorded above the CO molecule (red curve) and the bare Cu surface (black curve), positions marked in (a). The zero point of the $z$ axis defines the closest approach in the measurement, $z=100 \mathrm{pm}$ corresponds to the STM setpoint $V_{b}=-100 \mathrm{mV}$ and $\left\langle I_{t}\right\rangle=-100 \mathrm{pA}$ above the bare Cu surface. The COFI image has been recorded at $z_{\text {COFI. }}$ (c) Shortrange force vs distance $F_{\mathrm{SR}}(z)$ spectrum obtained by subtracting the $\Delta f(z)$ spectrum acquired above the $\mathrm{Cu}(110)$ surface [black in (b)] from the $\Delta f(z)$ spectrum acquired in the center of the $\mathrm{CO}$ molecule [red in (b)] and subsequent force deconvolution. ${ }^{34}$ (d) STM image of the copper oxide domain. Imaging parameters: $V_{b}=-100 \mathrm{mV}$ and $\left\langle I_{t}\right\rangle=-100 \mathrm{pA}$. The image has been processed with a $2 \times 2$ Gaussian lowpass filter. $^{35}$ (e) and (f) Constant-height $\Delta f$ images of the same area as in (d) recorded at $z=50 \mathrm{pm}$ and $z=0 \mathrm{pm}$, respectively. Far away (e), the AR-Cu atoms are imaged dark, and at closer distance (f), AR-O atoms are imaged bright. The overlaid structure marks positions of AR-Cu and AR-O atoms.
To obtain significant contrast in constant-height AFM images, the tip was then approached to the sample from the STM imaging height. The atomic features that are resolved first are depressions [Fig. 2(e)], which can be attributed to the AR-Cu atoms. ${ }^{15}$ The constantheight image in Fig. 2(e) is in excellent agreement with previously reported AFM images recorded of the $\mathrm{Cu}(110)-(2 \times 1) \mathrm{O}$ reconstruction with $\mathrm{CuOx}$ tips. ${ }^{15,16}$ Further approaching the surface leads to the emergence of increasing $\Delta f$ above the AR-O atomic sites [Fig. 2(f)], which is expected from short-range Pauli repulsion between the tip and the AR-O atoms (see calculated force vs distance curves in Ref. 15). The good agreement of our STM and AFM data with previous studies of Mönig et al. ${ }^{15,16}$ is further confirmation that the COFI image (a)

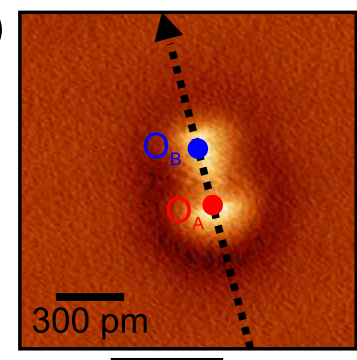

$-24.9 \square-0.4 \mathrm{~Hz}$
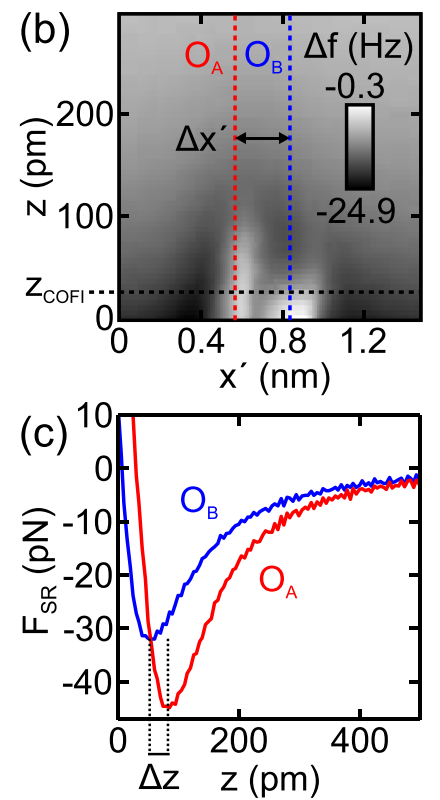

(d)

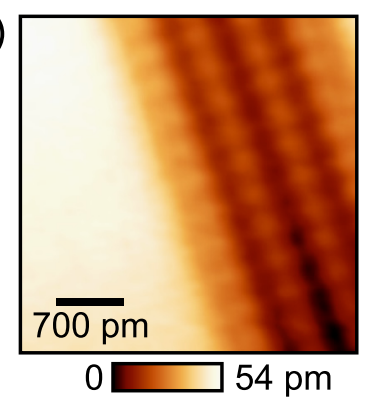

(e)

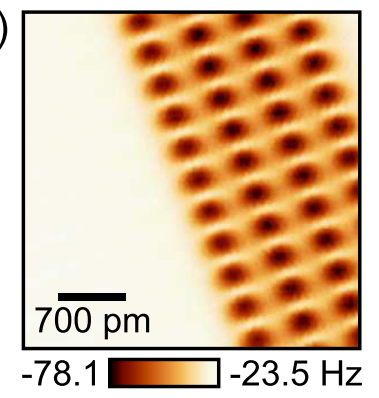

(f)

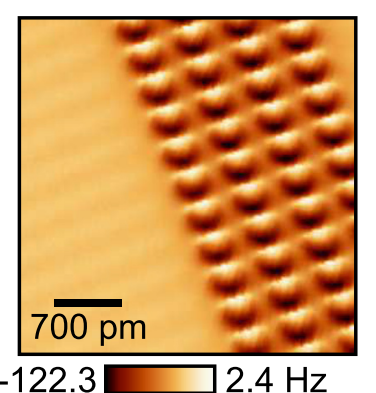

FIG. 3. (a) COFI image of a dimer CuOx tip. (b) $\Delta f\left(x^{\prime}, z\right)$ extracted along the dashed line in (a) from a set of $z$-dependent COFI images of the dimer $\mathrm{CuOx}$ tip. Vertical, dashed lines mark the positions of the two $O$ atoms $\left(O_{A}\right.$ and $\left.O_{B}\right)$ at the tip apex. The COFI image in (a) has been recorded at $z_{\mathrm{COFI}}$. (c) Short-range force vs distance $F_{\mathrm{SR}}(\mathrm{z})$ spectra recorded above the two $\mathrm{O}$ atoms, positions marked in (a) and (b), respectively. (d) STM feedback image of the $\mathrm{Cu}(110)-(2 \times 1) 0$ added-row reconstruction recorded with the dimer CuOx tip. Imaging parameters: $V_{b}=$ $-100 \mathrm{mV}$ and $\left\langle\mathrm{I}_{\mathrm{t}}\right\rangle=-100 \mathrm{pA}$. The image has been processed with a $2 \times 2$ Gaussian low-pass filter and z-drift corrected. ${ }^{35}$ (e) and (f) Constant-height $\Delta f$ images of the same area as in (d), recorded $190 \mathrm{pm}$ and $240 \mathrm{pm}$ closer to the surface with respect to the STM setpoint on the bare Cu surface, respectively. 
in Fig. 2(a) indeed corresponds to a $\mathrm{CuOx}$ tip with a single $\mathrm{O}$ atom at the apex [compare Fig. 1(a)].

In Fig. 3, we show experimental data for a tip with an apex consisting of more than one oxygen atom. The COFI image [Fig. 3(a)] shows two bright, repulsive features, which we attribute to two individual oxygen atoms at the tip apex. In the supplementary material, we show COFI images as a function of tip-sample distance for this tip to further illustrate this interpretation (Fig. S5). As the two $\mathrm{O}$ atoms are not at the same height above the plane of the surface, the COFI image has an associated tilt asymmetry. Figure 3(b) displays a vertical cut $\Delta f\left(x^{\prime}, z\right)$ extracted along the dashed line in Fig. 3(a) from a full set of $z$-dependent COFI images recorded with this tip. From this vertical cut, the lateral distance between the two $\mathrm{O}$ atoms at the tip apex was determined to be $\Delta x^{\prime}=250 \mathrm{pm}$. However, since the lateral deflection of the $\mathrm{CO}$ molecule leads to an increased lateral size of atomic features in AFM images, ${ }^{9}$ the value of $\Delta x^{\prime}$ determined here defines an upper boundary for the lateral offset between the two $\mathrm{O}$ atoms at the tip apex. Figure $3(\mathrm{c})$ shows $F_{\mathrm{SR}}(z)$ spectra recorded above the two $\mathrm{O}$ atoms, respectively. From the two spectra, short-range force minima of $-45 \mathrm{pN}\left(\right.$ atom $\mathrm{O}_{\mathrm{A}}$ ) and $-32 \mathrm{pN}\left(\right.$ atom $\mathrm{O}_{\mathrm{B}}$ ) are obtained, respectively, which are lower compared to the values for $\mathrm{CuOx}$ tips that have a single $\mathrm{O}$ atom at the apex. This can be attributed to the combined interaction of the two apex atoms with the CO molecule that cannot be separated using the on-off subtraction method. To estimate the vertical offset between the two $\mathrm{O}$ atoms at the tip apex, we compare the $z$ positions of the minima in $F_{\mathrm{SR}}(z)$ spectra $\left(z^{*}\right.$-method). ${ }^{38}$ Based on this method, we determined the vertical offset from the $F_{\mathrm{SR}}(z)$ spectra in Fig. 3(c) to be $\Delta z=35 \mathrm{pm}$. From the values obtained for $\Delta x^{\prime}$ and $\Delta z$, the distance between the $\mathrm{O}$ atoms is found to be $d=252 \mathrm{pm}$.

With this "dimer" CuOx tip, we recorded STM and AFM images of the $\mathrm{Cu}(110)-(2 \times 1) \mathrm{O}$ added-row reconstruction for tip fingerprinting as done in Ref. 15. The STM feedback image [Fig. 3(d)] is similar to the image recorded with the tip that had a single $\mathrm{O}$ atom at the apex. To obtain significant AFM contrast, the tip was again approached towards the surface from the STM imaging height. Note that the tip had to be moved closer to the surface than the single $\mathrm{O}$ atom tip. This can be attributed to the additional tunneling current contribution from the second $\mathrm{O}$ atom at the tip apex, which leads to a greater tip-sample distance with STM feedback on. ${ }^{20,21}$ Interestingly, at first, we see again the emergence of depressions [Fig. 3(e)], which can be attributed to attractive interaction between the tip and the AR$\mathrm{Cu}$ atoms. This appearance of the copper oxide domain is very similar to images that we recorded with tips that had a single $\mathrm{O}$ atom at the apex [compare Fig. 2(e)]. Only when the tip further approaches the sample [Fig. 3(f)], the images of the $\mathrm{Cu}(110)-(2 \times 1) \mathrm{O}$ added-row reconstruction exhibit an asymmetry due to the multi-atom tip apex that is clearly distinct from images recorded with the $\mathrm{CuOx}$ tip that had a single $\mathrm{O}$ atom at the apex: As compared to Fig. 2(f), the bright features that arise from Pauli repulsion between the tip and the AR-O atoms show a clear asymmetry. However, the determination of the exact tip structure based on the AFM images of the copper oxide domain would require theoretical modeling of such a dimer $\mathrm{CuOx}$ tip that has so far not been pursued.

This illustrates the danger of tip characterization with images of the oxide domain which show atomic resolution: Simply because individual atoms of the oxide can be resolved does not mean that the tip apex can be accurately determined, even when compared to simulated data. One attribute of the COFI method is to acquire data at a tip-sample height at which there is significant repulsive interaction between the tip and the adsorbate. This results in a very detailed and complete picture of the apex atoms responsible for AFM imaging. Of course, the effect of multiple atoms will also be seen on the oxide domain when acquiring data at smaller tipsample distances. However, it is more straightforward to directly determine the geometric positions of the apex atoms with an image of a point feature (a single CO molecule) vs that of a lattice. This is especially true for instances in which the spacing between apex atoms on the tip is comparable to that of the lattice, as in the example of our dimer tip.

In conclusion, we presented an efficient in-situ characterization of $\mathrm{CuOx}$ tips via the COFI technique and force spectroscopy. COFI images of $\mathrm{CuOx}$ tips terminating in a single $\mathrm{O}$ atom exhibit a circularly symmetric repulsive feature, very similar to images of CO-terminated tips on $\mathrm{Cu}(111)^{11}$ and $\mathrm{Cu}(110)$. Via force spectroscopy curves recorded with these single atom $\mathrm{CuOx}$ tips on $\mathrm{CO} / \mathrm{Cu}(110)$, we measured shortrange force minima ranging from $-15 \mathrm{pN}$ to $-30 \mathrm{pN}$, which is similar to data obtained for CO tips and illustrates the decreased reactivity of these tips as compared to bare metal tips. ${ }^{11,26}$ While AFM images of the $\mathrm{Cu}(110)-(2 \times 1) \mathrm{O}$ added-row reconstruction recorded with a tip terminating in two $\mathrm{O}$ atoms at tip-sample distances at which atomic resolution appears first in constant-height images showed similar features as tips terminating in only one $\mathrm{O}$ atom, the COFI image directly resolved the two-atom tip apex. Based on our findings, we suggest to in-situ characterize $\mathrm{CuOx}$ tips using COFI and force spectroscopy to unambiguously determine the chemical and structural compositions of the tip apex. Knowledge of these properties is of crucial importance, especially if the tips are used for experiments on other sample systems that are accompanied by theory, and the approach presented here provides an efficient way of tip characterization.

See supplementary material for line profiles extracted from the STM images of the copper oxide domain, COFI images of the CuOx tip as a function of tip-sample distance, $F_{\mathrm{SR}}(z)$-spectra for twelve $\mathrm{CuOx}$ tips, a COFI image and a $F_{\mathrm{SR}}(z)$-spectrum recorded with a COterminated tip on $\mathrm{CO} / \mathrm{Cu}(110)$, and COFI images of the dimer $\mathrm{CuOx}$ tip as a function of tip-sample distance.

We thank H. Mönig, A. Peronio, A. J. Weymouth, J. Berwanger, F. Huber, and S. Matencio for fruitful discussions and A. J. Weymouth and J. Berwanger for proofreading this manuscript. We are grateful to F. Pielmeier for the data measured with the $\mathrm{CO}$ tip on $\mathrm{Cu}(110)$. The authors acknowledge the Deutsche Forschungsgemeinschaft for funding within research Project No. CRC 1277, project A02.

\section{REFERENCES}

${ }^{1}$ G. Binnig, H. Rohrer, C. Gerber, and E. Weibel, Phys. Rev. Lett. 49, 57 (1982).

${ }^{2}$ G. Binnig, C. F. Quate, and C. Gerber, Phys. Rev. Lett. 56, 930 (1986).

${ }^{3}$ N. Moll, L. Gross, F. Mohn, A. Curioni, and G. Meyer, New J. Phys. 12, 125020 (2010).

${ }^{4}$ R. Temirov, S. Soubatch, O. Neucheva, A. Lassise, and F. S. Tautz, New J. Phys. 10, 053012 (2008).

${ }^{5}$ D. M. Eigler, C. P. Lutz, and W. E. Rudge, Nature 352, 600 (1991).

${ }^{6}$ L. Bartels, G. Meyer, and K.-H. Rieder, Appl. Phys. Lett. 71, 213 (1997).

${ }^{7}$ G. Kichin, C. Weiss, C. Wagner, F. S. Tautz, and R. Temirov, J. Am. Chem. Soc. 133, 16847 (2011). 
${ }^{8}$ L. Gross, F. Mohn, N. Moll, P. Liljeroth, and G. Meyer, Science 325, 1110 (2009).

${ }^{9}$ L. Gross, F. Mohn, N. Moll, B. Schuler, A. Criado, E. Guitián, D. Peña, A. Gourdon, and G. Meyer, Science 337, 1326 (2012).

${ }^{10}$ F. Mohn, B. Schuler, L. Gross, and G. Meyer, Appl. Phys. Lett. 102, 073109 (2013).

"7. Sun, M. P. Boneschanscher, I. Swart, D. Vanmaekelbergh, and P. Liljeroth, Phys. Rev. Lett. 106, 046104 (2011).

${ }^{12}$ A. J. Weymouth, T. Hofmann, and F. J. Giessibl, Science 343, 1120 (2014).

${ }^{13}$ P. Hapala, G. Kichin, C. Wagner, F. S. Tautz, R. Temirov, and P. Jelínek, Phys. Rev. B 90, 085421 (2014).

${ }^{14}$ N. Moll, B. Schuler, S. Kawai, F. Xu, L. Peng, A. Orita, J. Otera, A. Curioni, M. Neu, J. Repp, G. Meyer, and L. Gross, Nano Lett. 14, 6127 (2014).

${ }^{15}$ H. Mönig, D. R. Hermoso, O. Díaz Arado, M. Todorović, A. Timmer, S. Schüer, G. Langewisch, R. Pérez, and H. Fuchs, ACS Nano 10, 1201 (2016).

${ }^{16} \mathrm{H}$. Mönig, S. Amirjalayer, A. Timmer, Z. Hu, L. Liu, O. Díaz Arado, M. Cnudde, C. A. Strassert, W. Ji, M. Rohlfing, and H. Fuchs, Nat. Nanotechnol. 13, 371 (2018).

${ }^{17}$ O. E. Dagdeviren, Phys. Rev. Applied 11, 024068 (2019).

${ }^{18}$ J. Bamidele, Y. Kinoshita, R. Turanský, S. H. Lee, Y. Naitoh, Y. J. Li, Y. Sugawara, I. Štich, and L. Kantorovich, Phys. Rev. B 86, 155422 (2012).

${ }^{19}$ J. Bamidele, Y. Kinoshita, R. Turanský, S. H. Lee, Y. Naitoh, Y. J. Li, Y. Sugawara, I. Štich, and L. Kantorovich, Phys. Rev. B 90, 035410 (2014).

${ }^{20}$ M. Z. Baykara, M. Todorović, H. Mönig, T. C. Schwendemann, Ö. Ünverdi, L. Rodrigo, E. I. Altman, R. Pérez, and U. D. Schwarz, Phys. Rev. B 87, 155414 (2013).

${ }^{21}$ H. Mönig, M. Todorović, M. Z. Baykara, T. C. Schwendemann, L. Rodrigo, E. I. Altman, R. Pérez, and U. D. Schwarz, ACS Nano 7, 10233 (2013).

${ }^{22}$ J. Welker and F. J. Giessibl, Science 336, 444 (2012).
${ }^{23}$ J. Welker, A. J. Weymouth, and F. J. Giessibl, ACS Nano 7, 7377 (2013).

${ }^{24} \mathrm{M}$. Emmrich, F. Huber, F. Pielmeier, J. Welker, T. Hofmann, M. Schneiderbauer, D. Meuer, S. Polesya, S. Mankovsky, D. Ködderitzsch, H. Ebert, and F. J. Giessibl, Science 348, 308 (2015).

${ }^{25}$ Y. Sugimoto, P. Pou, M. Abe, P. Jelinek, R. Pérez, S. Morita, and Ó. Custance, Nature 446, 64 (2007).

${ }^{26}$ T. Hofmann, F. Pielmeier, and F. J. Giessibl, Phys. Rev. Lett. 112, 066101 (2014).

${ }^{27}$ F. J. Giessibl, Appl. Phys. Lett. 76, 1470 (2000).

${ }^{28}$ T. R. Albrecht, P. Grütter, D. Horne, and D. Rugar, J. Appl. Phys. 69, 668 (1991).

${ }^{29}$ D. J. Coulman, J. Wintterlin, R. J. Behm, and G. Ertl, Phys. Rev. Lett. 64, 1761 (1990).

${ }^{30}$ K. Kern, H. Niehus, A. Schatz, P. Zeppenfeld, J. Goerge, and G. Comsa, Phys. Rev. Lett. 67, 855 (1991).

${ }^{31} \mathrm{~J}$. Harl and G. Kresse, Surf. Sci. 600, 4633 (2006).

${ }^{32}$ S. Kishimoto, M. Kageshima, Y. Naitoh, Y. J. Li, and Y. Sugawara, Surf. Sci. 602, 2175 (2008).

${ }^{33}$ P. Hofmann, K. Schindler, S. Bao, V. Fritzsche, A. M. Bradshaw, and D. Woodruff, Surf. Sci. 337, 169 (1995).

${ }^{34}$ J. E. Sader and S. P. Jarvis, Appl. Phys. Lett. 84, 1801 (2004).

${ }^{35}$ I. Horcas, R. Fernández, J. M. Gómez-Rodríguez, J. Colchero, J. GómezHerrero, and A. M. Baro, Rev. Sci. Instrum. 78, 013705 (2007).

${ }^{36}$ M. Ternes, C. González, C. P. Lutz, P. Hapala, F. J. Giessibl, P. Jelínek, and A. J. Heinrich, Phys. Rev. Lett. 106, 016802 (2011).

37J. E. Sader, B. D. Hughes, F. Huber, and F. J. Giessibl, Nat. Nanotechnol. 13, 1088 (2018).

${ }^{38}$ B. Schuler, W. Liu, A. Tkatchenko, N. Moll, G. Meyer, A. Mistry, D. Fox, and L. Gross, Phys. Rev. Lett. 111, 106103 (2013). 